\title{
Effects of Expression of Mammalian G $\alpha$ and Hybrid Mammalian- Yeast G $\alpha$ Proteins on the Yeast Pheromone Response Signal Transduction Pathway
}

\author{
YOON-SE KANG, ${ }^{1} \dagger$ JEFFREY KANE, ${ }^{1}$ JANET KURJAN $^{2}{ }^{2}$ JEFFREY M. STADEL, ${ }^{3}$ AND DONALD J. TIPPER ${ }^{1 *}$ \\ Department of Molecular Genetics and Microbiology, University of Massachusetts Medical Center, Worcester, \\ Massachusetts 01655'; Department of Biological Sciences, Columbia University, New York, New York $10027^{2}$; \\ and Pharmacology Department, SmithKline Beecham Pharmaceuticals, King of Prussia, Pennsylvania 19406 ${ }^{3}$
}

Received 7 December 1989/Accepted 27 February 1990

\begin{abstract}
Scg1, the product of the Saccharomyces cerevisiae SCG1 (also called GPA1) gene, is homologous to the $\alpha$ subunits of $G$ proteins involved in signal transduction in mammalian cells. Scg1 negatively controls the pheromone response pathway in haploid cells. Either pheromonal activation or an scg1 null mutation relieves the negative control and leads to an arrest of cell growth in the G1 phase of the cell cycle. Expression of rat Gos was previously shown to complement the growth defect of scg1 null mutants while not allowing mating. We have extended this analysis to examine the effects of the short form of Gas (which lacks 15 amino acids present

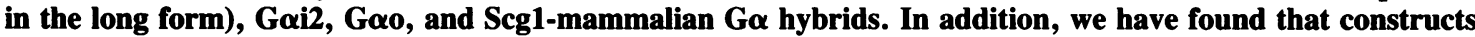
able to complement scgl are also able to inhibit the response to pheromone and mating when expressed in a wild-type SCG1 strain. Overexpression of Scg1 has a similar inhibitory effect. These results are consistent with a model proposed for the action of Scg1 as the $\alpha$ component of a heterotrimeric $G$ protein in which the $\beta \gamma$ component (Ste4/Ste18) activates the pheromone response after dissociation from Scg1. They suggest that the G $\alpha$ constructs able to complement scg1 can interact with $\beta \gamma$ to prevent activation of the pathway but are unable to interact with pheromone receptors to activate the pathway.
\end{abstract}

The $\mathbf{G}$ protein family transduces hormonal and sensory signals received by a major class of transmembrane receptors in eucaryotic cells. These receptors respond to an extracellular signal by directly activating a $G$ protein on the cytoplasmic face of the membrane (for a review, see references 12, 26, and 29). Mammalian $G$ proteins are heterotrimers of $\alpha$ (39 to 52 kilodaltons), $\beta$ (35 to 36 kilodaltons), and $\gamma$ (8 to 10 kilodaltons) subunits. The $\alpha$ subunits have highly conserved regions that are similar to the guanine nucleotidebinding pockets of the structurally defined GTP-binding proteins, EF-Tu and c-H-ras $(9,31)$. In the unstimulated state, the nucleotide-binding domain of $\alpha$ is occupied by GDP, and the G protein exists as a stable, membrane-bound heterotrimer. Interaction of an agonist with the appropriate receptor induces a receptor- $G$ protein interaction that results in replacement of GDP by GTP and dissociation of $\alpha$ (GTP) from $\beta \gamma$. In the phototransduction and $\beta$-adrenergic systems, $\alpha($ GTP) subunits directly activate effectors; however, recent evidence suggests that $\beta \gamma$ can stimulate phospholipase $A_{2}$ activity in cardiac cells $(4,17)$.

In the yeast Saccharomyces cerevisiae, haploid cells of a and $\alpha$ mating types are prepared for mating by mutual interactions mediated by peptide pheromones (for a review, see reference 8 ). $\alpha$ cells secrete $\alpha$-factor, a tridecapeptide that binds to the $\alpha$-factor receptor present on a cells, and a cells secrete a-factor, a modified dodecapeptide that binds to the a-factor receptor present on $\alpha$ cells. Binding of either pheromone to its receptor results in an arrest of the cell cycle in $\mathrm{G} 1$ and other responses that are necessary for mating. Sequencing of the structural genes for the a- and $\alpha$-factor

\footnotetext{
* Corresponding author.

$\dagger$ Present address: Department of Biochemistry, College of Medicine, Gyeong-Sang National University, Chinju 620, Republic of Korea.
}

receptors (STE3 and $S T E 2$, respectively; 5, 13, 25) suggests that both have the seven transmembrane-spanning domain topology characteristics of mammalian $G$ protein-coupled receptors.

Genes involved in pheromone response and mating that encode $\alpha, \beta$, and $\gamma$ homologs have been identified. The $\alpha$ homolog (Scg1) is encoded by SCGI (10) (also called GPA1; $22,23)$, and the $\beta$ and $\gamma$ subunits (Ste4, Ste18) are encoded by STE4 and STE18, respectively (32). Null mutations in these genes result in phenotypes in haploids but not diploids. The ste 4 and ste 18 null mutants are sterile (i.e., are defective in pheromone response and mating), indicating that wildtype Ste4 and Ste18 play positive roles in activation of the pheromone response pathway. The $s c g 1$ null mutants show cell cycle arrest and morphological changes characteristic of pheromone response; this phenotype represents constitutive activation of the pheromone response pathway, indicating that wild-type Scg1 plays a negative or inhibitory role (10, 14, 22, 24).

Genetic results indicate that $\beta$ and $\gamma$ act downstream of $\alpha$ in the pathway $(1,24,32)$. A model consistent with these results suggests that, in the resting state, the $\mathrm{G}$ protein exists as a heterotrimer, $\alpha(G D P) \beta \gamma(10,14,32)$. After activation of the pathway and guanine nucleotide exchange, $\alpha($ GTP) and $\beta \gamma$ dissociate, and $\beta \gamma$ stimulates pheromone response by activation of a downstream effector. The negative role of $\alpha$ results from inhibition of $\beta \gamma$ activity.

It was previously shown that expression of rat G $\alpha$ s (the $\alpha$ subunit of the $G$ protein that activates adenylate cyclase) under the control of the yeast $C U P I$ promoter complemented the growth and morphological defects of an scgl null mutant (10). The $s c g l[\mathrm{G} \alpha \mathrm{s}]$ cells were sterile. In this paper, we have extended the analysis of the complementation of $s c g l$ by mammalian $\mathrm{G} \alpha$ genes to $\mathrm{G} \alpha \mathrm{i} 2$ (one of three related $\mathrm{G} \alpha$ proteins involved in inhibition of adenylate cyclase) and 
TABLE 1. Yeast strains

\begin{tabular}{|c|c|c|}
\hline Strain & $\begin{array}{l}\text { Genotypic }{ }^{a} \text { or phenotypic } \\
\text { characteristics }\end{array}$ & $\begin{array}{l}\text { Source or } \\
\text { reference }\end{array}$ \\
\hline W303 & $\begin{array}{l}\text { MATa } / M A T \alpha[\text { ade2-1 his3-11,5 } \\
\text { leu2-3,112 trp1-1 ura3-1 canl-100] }\end{array}$ & R. Rothstein \\
\hline D109 & $\begin{array}{l}\text { SCG1/scg1::URA3 derivative of } \\
\text { W303 }\end{array}$ & 10 \\
\hline D111 & $\begin{array}{l}S C G 1 / \text { scg } 1:: L a c Z-[L E U 2] \text { deriva- } \\
\text { tive of } \mathrm{W} 303\end{array}$ & 10 \\
\hline $381 G$ & $\begin{array}{l}\text { MATa cryl ade2 his } 4^{\mathrm{am}} \text { lys } 2 \text { trpl } \\
\text { tyrl SUP } \text { la }^{\mathrm{am}}\end{array}$ & L. Hartwell \\
\hline DJ147-1-1 & $\begin{array}{l}\text { MAT } \alpha \text { ura3-52, otherwise isogenic } \\
\text { to } 381 \mathrm{G}\end{array}$ & D. Jenness \\
\hline DJ147-1-2 & $\begin{array}{l}\text { MATa ura3-52 leu2-3,112 } \mathrm{Tyr}^{+} \\
\text {otherwise isogenic to } 381 \mathrm{G}\end{array}$ & D. Jenness \\
\hline DJ211-3-4 & barl-1 derivative of $147-1-2$ & D. Jenness \\
\hline
\end{tabular}

${ }^{a}$ Markers shown in brackets are homozygous.

Gao (a $\mathrm{G}$ protein of unknown function abundant in brain tissue). We have also constructed and tested hybrid Scg1 and mammalian $\mathrm{G} \alpha$ genes. We show that expression of many of these constructs efficiently complements the scgl growth defect but does not allow mating. In addition, their expression exerts a dominant inhibitory effect on the response to $\alpha$-factor and on mating in both a and $\alpha$ cells. Overexpression of $S C G 1$ has the same inhibitory effect.

\section{MATERIALS AND METHODS}

Yeast strains, transformations, and expression vectors. The yeast strains used are described in Table 1. Transformants of these strains with the plasmid constructs described below were selected by uracil or tryptophan prototrophy and maintained on SC-Ura medium (synthetic complete medium lacking uracil; 3) or SC-Trp medium. All media contained 2\% glucose except media designed for induction of pGAL constructs, which contained $2 \%$ sucrose and $1 \%$ galactose. YEP-Gal medium, used for tetrad analysis, is yeast extractpeptone (3) containing $2 \%$ galactose. All plasmid constructs were propagated in Escherichia coli AKE28 (20) under ampicillin selection.

Expression vectors, pPGK (YEpDT-PGK; Fig. 1). The segment comprising $\mathrm{Amp}^{\mathrm{R}}$, $\mathrm{Ori}^{\mathrm{E}}$, and $U R A 3$ was derived from YIp5. The 2- $\mu \mathrm{m}$ replication origin (a 1,424-base-pair (bp) HaeIII-to-HpaI fragment from YEp24) was inserted at the EcoRI site of YIp5, as in p1A1 (3). The PGK expression cassette was inserted between the ClaI and NruI sites (converted by linker insertion to XhoI and SalI, respectively) and consisted of two fragments derived from pMA91 (20). pMA91 contained a 2.9-kilobase HindIII fragment of yeast DNA comprising the $P G K$ gene and flanking sequences and a deletion which joined nucleotide -2 to the $B$ III site 30 codons from the $\mathrm{C}$ terminus of the gene. The 672-bp fragment comprising $\mathrm{UAS}_{\mathrm{PGK}}$ was derived by inserting an XhoI linker at the DraI site at -670 and an EcoRI linker at the BglII site of pMA91 at -2 . The 300-bp translation and transcription termination fragment was derived by $B a l 31$ recision at the $B g I I I$ site followed by insertion of a $B a m H I$ linker one codon upstream of the terminator codon (TAA) in the PGK reading frame. Terminators occurred in all three reading frames within $22 \mathrm{bp}$ of the BamHI site, immediately followed by a ClaI site (GGATCCGGGA AATAAATTGAATTGAATTGAAATGCAT). A Sall linker was inserted at the HindIII site 300 bp from this BamHI site, downstream of the $P G K$ transcription terminator. Filling in of the two PGK fragments cut at the their EcoRI and BamHI sites, followed by religation with a HindIII linker, regenerated both sites (GAATTCCAAGCTTGGGATCC), producing YEpDT-PGK with unique EcoRI, HindIII, and BamHI cloning sites for expression (Fig. 1).

pGAL (YCpDT-GAL; Fig. 1). The 672-bp XhoI-EcoRI fragment comprising $\mathrm{UAS}_{\mathrm{PGK}}$ in $\mathrm{pPGK}$ was replaced with the 685-bp BamHI-EcoRI UAS GAL $_{\text {fragment from pBM272 }}$ (provided by Mark Johnston) after filling in both XhoI and BamHI sites. The XhoI site was regenerated. The product was YEpDT-GAL. The 985-bp XhoI-Sall fragment of YEpDT-GAL comprising the expression cassette was reinserted into pBM272 between the EcoRI and SalI sites after filling in both the EcoRI and XhoI sites, producing YCpDTGAL (Fig. 1). This vector contained the ARS1, CEN4, and $U R A 3$ yeast fragments (indicated in Fig. 1) derived from pBM272.

pCUP (pYSK-136; Fig. 1). The pCUP vector (11) comprised the $A m p^{R}$ and Ori fragments of $\mathrm{pBR} 322$, the 2kilobase EcoRI origin fragment of $2 \mu \mathrm{m}$, a 900-bp TRPI fragment, and an expression cassette consisting of a 430-bp UAS $_{\text {CUP1 }}$ fragment and a 310-bp CYC1 terminator fragment separated by unique $N c o$ I and $A s p 718 \mathrm{I}$ expression sites.

cDNAs and expression of full-length constructs. $\mathbf{\alpha} \boldsymbol{\alpha} \mathbf{c D N A s}$. Rat $G \alpha$ cDNAs were provided by Randall $R$. Reed (15) as EcoRI fragments in $\lambda$ phage or by Gary Johnson as sub-
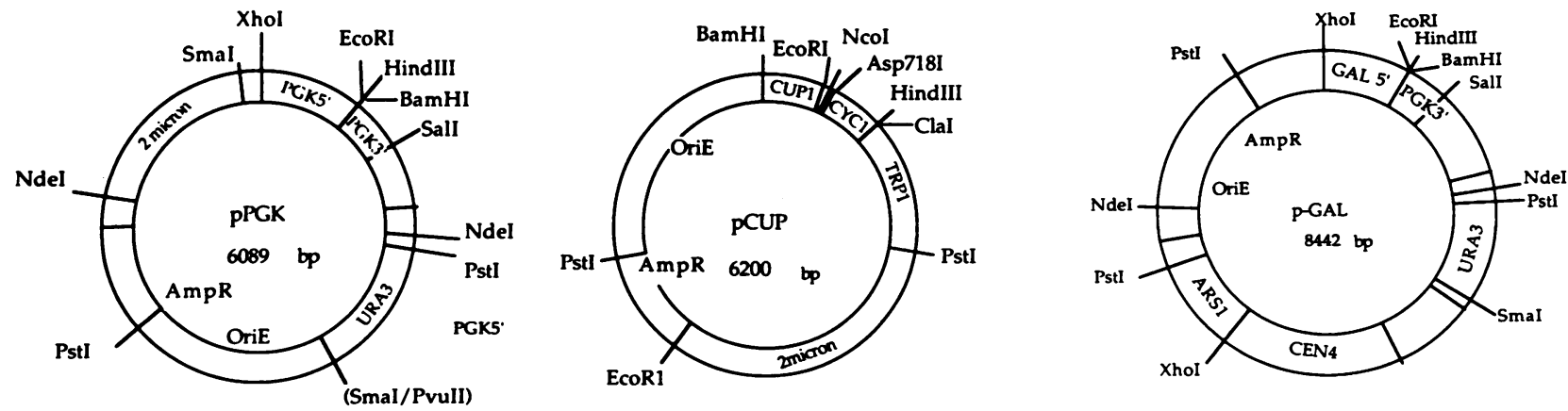

FIG. 1. Expression vectors. pCUP (pYSK; 11) contains an expression cassette consisting of the CUPl promoter and $C Y C 1$ transcription terminator. pGAL contains an expression cassette consisting of the $G A L 1,10$ promoter and the $P G K$ transcription terminator. pPGK contains an expression cassette consisting of the $P G K$ promoter and transcription terminator sequence. $G \alpha$ gene fragments were inserted into the unique EcoRI, HindIII, or BamHI sites in pPGK and pGAL or at the NcoI site in pCUP. All three vectors contained the pBR322 Amp ${ }^{\mathrm{R}}$ gene and replication origin and the indicated yeast sequences (see Materials and Methods). 2 micron, $2 \mu \mathrm{m}$. 


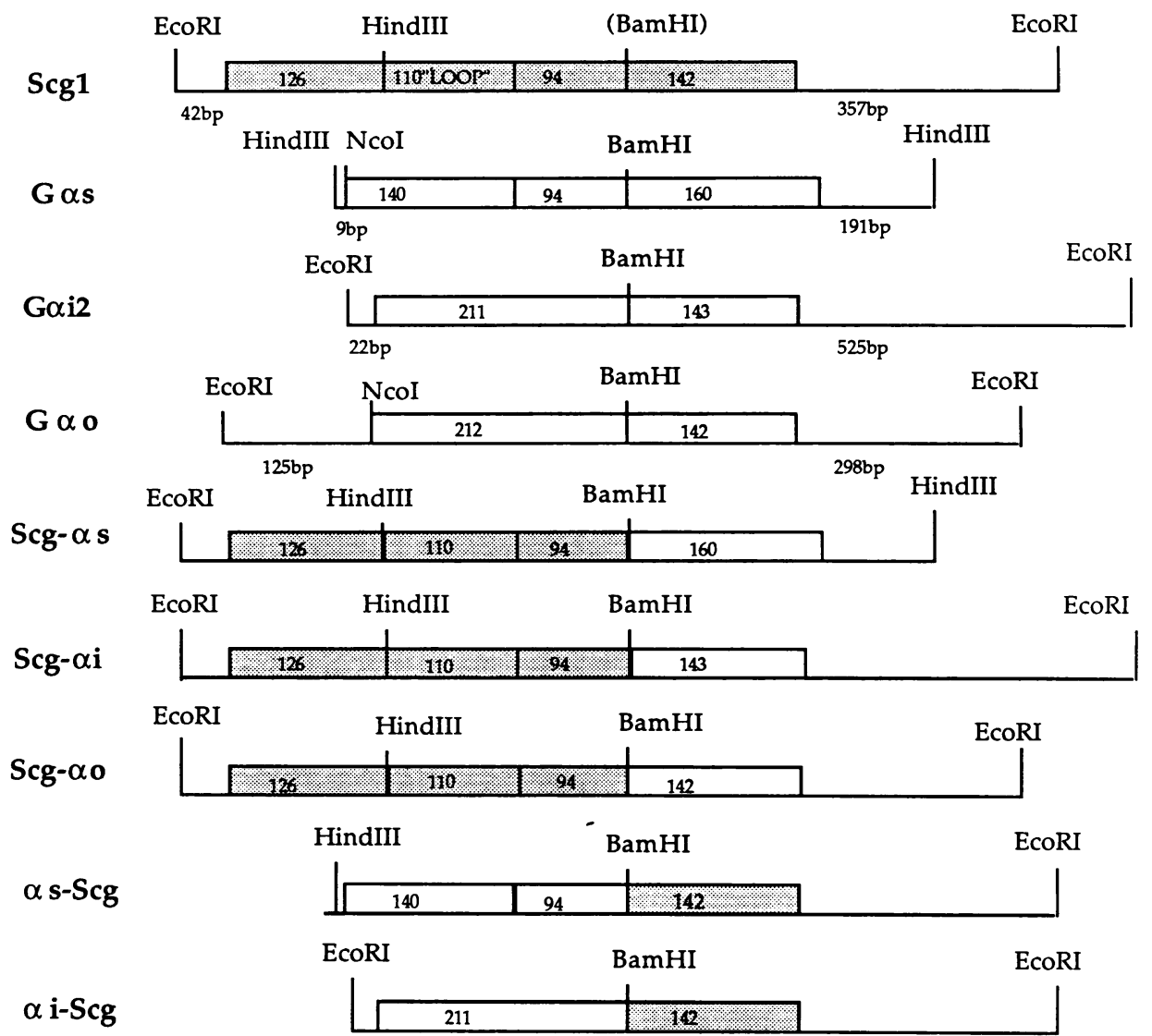

FIG. 2. G $\alpha$ proteins and fusions: open reading frames with the lengths (in amino acid residues) of various regions indicated. Symbols: $S C G 1 ; \square$, mammalian G $\alpha$ cDNAs. The HindIII site in SCGl is approximately at the start of the unique 110-residue loop in Scg1 which has no counterpart in the mammalian G $\alpha$ proteins $(10,23)$. A BamHI site was introduced into $S C G I$ at a position homologous to a BamHI site in $\mathrm{G} \alpha \mathrm{s}, \mathrm{G} \alpha \mathrm{i} 2$, and $\mathrm{G} \alpha \mathrm{o}$ (see Discussion), and the hybrid genes shown were constructed. The sizes (in base pairs) of the 5' and 3' sequences included when these constructs were inserted in the pGAL and pPGK expression vectors (Fig. 1) are also indicated. The pCUP constructs utilized an NcoI site at the G $\alpha$ ATG initiation codon; therefore, no rat 5' sequences were present upstream of the initiation codon in these constructs (data not shown).

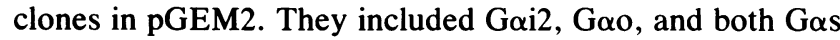

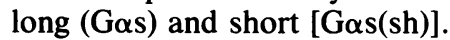

pCUP expression constructs. The Gas cDNA clone was cleaved at the $A f$ III site 27 bp past the translation termination codon. After being filled in with DNA polymerase (Klenow fragment) and cut with $N$ coI at the initiation codon (boldface) (CCATGG), the 1,218-bp fragment of G $\alpha$ s was cloned into pCUP between the NcoI and filled-in Asp718I sites. The $\mathrm{G} \alpha \mathrm{s}(\mathrm{sh})$ construct was made by exchanging the internal BamHI fragment of Gos in pCUP- $\alpha$ s for the equivalent fragment of $G \alpha s(s h)$. Similar procedures were used to insert

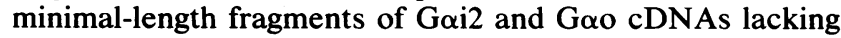
rat 5' sequences into the same expression sites.

pGAL and pPGK expression constructs (Fig. 2). To insert rat $\mathrm{G} \alpha$ s into pPGK, the pGEM clone was cleaved at the unique $N$ coI site at the translation start point. Insertion of a 10-bp HindIII linker after filling in produced a HindIII site at -9 , leaving only $1 \mathrm{bp}$ of rat $5^{\prime}$ sequence before the initiation codon (CCAAGCTTGGCATG). The 1,374-bp HindIII fragment starting at this point and ending $191 \mathrm{bp}$ downstream of the translation terminator for $G \alpha s$ was inserted into the HindIII sites of pPGK and pGAL for expression.

Rat Goi2 cDNA was provided as a 1,750-bp EcoRI fragment in pGEM2. pPGK- $\alpha \mathrm{i}$ was produced by partial $B g l \mathrm{I}$ digestion of the pGEM2 clone followed by treatment with the Klenow fragment of DNA polymerase and insertion of a 10-bp EcoRI linker, producing an EcoRI site at -22 and retaining $14 \mathrm{bp}$ of rat $5^{\prime}$ sequence (CCGAATTCGGCGG CGGACGGCAGGATG). The 1,619-bp EcoRI fragment from this site to the EcoRI site 525 bp downstream of the translation terminator for Gai2 was inserted into the EcoRI sites of pPGK and pGAL for expression (Fig. 2).

The yeast $S C G I$ gene was cloned into M13 mp10 as a 1,931-bp EcoRI fragment. Mutagenesis with the oligonucleotide 5'-TTAGCATCACATCAAGAATTCAGAG-3' (underlined nucleotides indicate positions resulting in nucleotide changes) was employed to insert an EcoRI site at -42 (Fig. 2). The new 1,762-bp EcoRI fragment was cloned into M13 mp19 and mutated with 5'-GAAGTGGATCCATTGTT TCG $-3^{\prime}$ to create a silent $\mathrm{BamHI}$ site at +989 , producing Scg1(B). The 1,762-bp EcoRI fragment, with and without this mutation, was cloned into pGAL for expression, producing pGAL-Scg and pGAL-Scg(B) (Fig. 2). pPGK-Scg was constructed similarly.

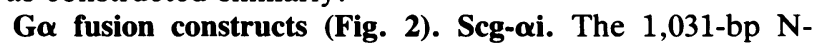
terminal fragment of SCGI from the EcoRI site at -42 to the mutant BamHI site at 989 and the 1,018-bp C-terminal fragment of Goi2 from the BamHI site at 634 to the downstream HindIII site of the pGEM 2 multicloning site were inserted simultaneously into pGAL and also into pPGK, both cut with EcoRI and HindIII. The sizes of the protein 
fragments in the hybrids resulting from this and other fusions are indicated in Fig. 2.

Scg-as. The same SCGl fragment and the 666-bp Cterminal fragment of $\mathrm{G} \alpha$ s from the BamHI site at 707 to the downstream HindIII site were inserted into the expression vectors as described above.

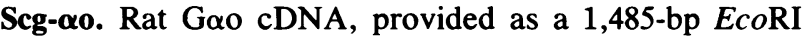
fragment in pGEM2, was cloned directly into the EcoRI site of pPGK, producing pPGK- $\alpha$. This construct had $116 \mathrm{bp}$ of 5'- and 298 bp of $3^{\prime}$-flanking cDNA sequence (Fig. 2). The fragment of Goo from the BamHI site at 635 to the BamHI site just downstream of the $E c o$ RI site in pPGK- $\alpha$ o (Fig. 1) was isolated and used to replace the $B a m H I$ fragment from 989 to the same vector site in pPGK-Scg(B), producing pPGK-Scg- $\alpha$ o (Fig. 2).

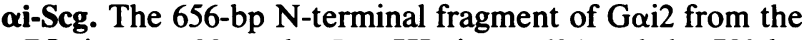
EcoRI site at -22 to the BamHI site at 634 and the $783-b p$ C-terminal fragment of $S C G 1$ from the mutant BamHI site at 989 to the downstream HindIII site were inserted into expression vectors simultaneously, as described above.

as-Scg. The 710-bp N-terminal fragment of G $\alpha$ s from the HindIII site at -9 to the second BamHI site was obtained by partial BamHI hydrolysis and cloned into the pPGK and pGAL vectors cleaved with $H$ indIII and BamHI. The 752-bp C-terminal SCGI BamHI fragment from 989 to the downstream BamHI site was subsequently inserted in the correct orientation to create the $\alpha s-S c g$ fusions.

Assays for effects on the pheromone response pathway. Complementation of the growth defect due to scgl null mutations was tested by transformation of strain D109 or D111 (Table 1) and sporulation and dissection of the diploids. Diploids containing pGAL constructs were dissected on YEP-Gal medium, diploids containing pCUP constructs were dissected on SC-Trp $+\mathrm{Cu}(100 \mu \mathrm{M})$ medium, and diploids containing the constitutive pPGK constructs were dissected on SC-Ura medium. Tetrads were monitored for growth of more than two spores per tetrad, and viable segregants were picked and tested for the marker present at scgl and for mating.

Response to $\alpha$-factor was tested by monitoring growth inhibition. Transformants of strain DJ211-3-4 (Table 1) were plated as lawns in soft agar overlays on SC-Ura medium ( $\mathrm{pH}$ 4.7) with appropriate carbon sources. Disks containing different quantities of $\alpha$-factor $\left(10 \mu\right.$ l of $0 \times 10^{-6}, 2 \times 10^{-6}, 5$ $\times 10^{-6}$, and $\left.10 \times 10^{-6} \mathrm{M}\right)$ were placed on the lawns. After 24 and $48 \mathrm{~h}$ at $30^{\circ} \mathrm{C}$, zones of growth inhibition surrounding the disks were inspected for diameter and density of growth compared with the rest of the lawn. Clear, sharp-edged zones whose diameters were proportional to the log of $\alpha$-factor concentration were produced on controls containing the vectors without inserts. Constructs that inhibited pheromone response allowed some growth within the $\alpha$-factor zones; estimates of growth density were made visually. Repetition with multiple independent transformants gave reproducible results.

Inhibition of mating was tested by quantitative mating assays. Transformants of strains DJ147-1-1 and DJ147-1-2 (Table 1) were grown to mid-exponential phase on SC-Ura medium ( $\mathrm{pH}$ 4.7) containing appropriate carbon sources, and mating efficiency was assayed by a membrane filter procedure (1).

\section{RESULTS}

Expression of heterologous and hybrid Ga proteins in yeast. In mammalian cells, major forms of $\mathrm{G} \alpha \mathrm{s}$ are expressed that differ by the presence or absence of 15 amino acids due to differential splicing (12). The form previously shown to complement the growth and morphological defects of scgl null mutants was $G \alpha$ s under the control of the copperinducible $C U P I$ promoter (10). We have extended this

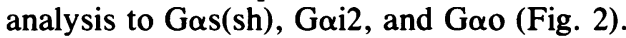

To examine the functional aspects of $\mathrm{G} \alpha$ protein domains, hybrids were made between $S C G 1$ and rat $\mathrm{G} \alpha$ cDNAs (diagrammed in Fig. 2) as described in Materials and Methods. Construction of these hybrids involved introducing a silent mutation in the Ile-coding codon 331 of $S C G l$ that introduces a BamHI site to produce $S C G l(B)$. BamHI sites were present in $\mathrm{G} \alpha \mathrm{s}, \mathrm{G} \alpha \mathrm{o}$, and $\mathrm{G} \alpha \mathrm{i} 2$ at the analogous position and were used to construct in-frame fusions between $S C G l$ and the three rat $\mathrm{G} \alpha \mathrm{cDNA}$ genes.

The heterologous and hybrid $\mathrm{G} \alpha$ proteins were expressed in yeast by using three different vectors, pCUP, pGAL, and pPGK (Fig. 1). To allow efficient expression, the heterologous or hybrid gene in each of the plasmids was flanked by a yeast upstream regulatory region and a yeast terminator. pGAL is a YCp (stable, low-copy-number) vector with the galactose-inducible $G A L 1,10$ promoter and $P G K$ terminator. pPGK is a YEp (high-copy-number) vector with the constitutive $P G K$ promoter and the $P G K$ terminator. pCUP is a YEp vector with the copper-inducible $C U P 1$ promoter and the $C Y C l$ terminator.

The differences in plasmid copy number and promoter strength should have resulted in different expression levels for each of the vectors. Relative steady-state levels of transcripts, as determined by Northern (RNA) blot analysis with appropriate probes for the heterologous $\mathrm{G} \alpha$ proteins, were estimated to be 1:50:250 for pGAL, pPGK, and pCUP constructs, respectively (data not shown). The induced pGAL-Scg transcript in strain DJ211-3-4 was present at about twice the level of the endogenous 1,700 -bp transcript derived from the wild-type gene (data not shown).

A polyclonal antiserum raised against a fusion of $G \alpha$ s to the N-terminal 81 residues of the influenza virus NSI protein (34) was used to detect total cellular Gos proteins by Western blot (immunoblot) (data not shown). Protein was undetectable in cells of strain DJ211-3-4 expressing pGAL$\alpha s$. Proteins of the expected sizes were detected in cells expressing pCUP- $\alpha s$, pPGK- $\alpha s, \mathrm{pPGK}-\alpha s-\mathrm{Scg}$, and pPGKScg- $\alpha$ s. All were detected at similar levels in spite of the higher transcript levels expressed by pCUP- $\alpha$ s. For some constructs (e.g., pCUP- $\alpha$ s and pPGK- $\alpha \mathrm{s}$ ), therefore, protein and RNA levels did not correlate well. Protein levels in cells expressing pCUP- $\alpha \mathrm{s}(\mathrm{sh})$ were 5- to 10-fold higher than for pCUP- $\alpha$ s.

Complementation of scg1 null mutations. In haploid yeast, scgl null mutations result in a growth arrest due to constitutive activation of the pheromone response pathway $(10$, 14, 24). The scgl spores that arise from dissection of an SCGI/scgl diploid form microcolonies (see Fig. 3; tetrads with vector alone). It was previously shown that expression of pCUP- $\alpha$ s in scgl spores resulted in spore colonies that were smaller than those of the wild type (10; see Fig. 3) but that growth was similar to that of the wild type after the colonies were picked to fresh plates. The reason for the initial growth lag is unclear; however, the size of $s c g l$ spore colonies expressing the various $\mathrm{G} \alpha$ constructs provided a measure of complementation of the scgl growth defect. The ability of mammalian $\mathrm{G} \alpha$ genes and hybrids between the $S C G I$ and mammalian genes to complement $s c g l$ was tested by transformation of $S C G 1 / s c g 1$ diploids with the various constructs and examination of the growth and mating of 


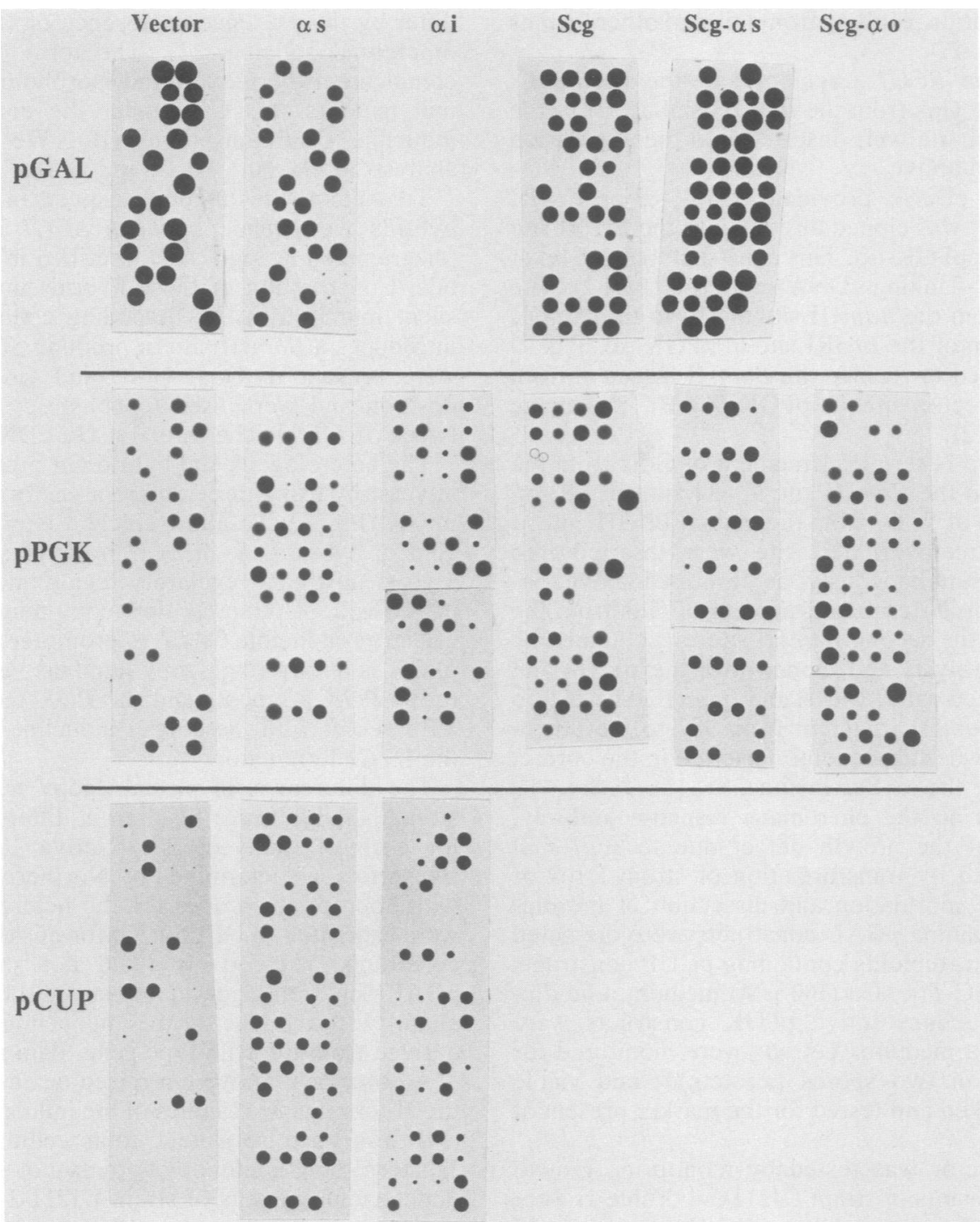

FIG. 3. Complementation of $s c g 1$ growth defect by G $\alpha$ constructs. Strain D111 (SCG1/scgl) containing the indicated constructs was sporulated and dissected onto medium that provided the appropriate selection and induction of the various plasmid constructs (see Materials and Methods). With vector alone, tetrads gave rise to two wild-type colonies and two barely visible colonies. The larger colony size for more than two spores per tetrad indicates complementation of the scgl growth defect. The relative complementation by these constructs is summarized in Table 2.

spores obtained by sporulation of the diploids and tetrad analysis. Representative tetrads are shown in Fig. 3.

All of the $\mathrm{G} \alpha$ s constructs complemented the $s c g 1$ growth defect, but none allowed mating (Fig. 3 and Table 2). Complementation was poorest with $\mathrm{pGAL}-\alpha \mathrm{s}$, intermediate with pCUP- $\alpha \mathrm{s}$, and best with pPGK- $\alpha \mathrm{s}$. Expression of Gos(sh) under the control of the CUPI promoter [pCUP$\alpha s(\mathrm{sh})$ ] produced results indistinguishable from those produced with the long form (pCUP- $\alpha$; Fig. 3).

Gai2 expressed from pCUP or pPGK but not from pGAL weakly complemented the scgl growth defect (Fig. 3 and Table 2). Again, pPGK-Goi2 gave the strongest effect. The weakly complemented cells were sterile. No complementation of scgl was seen in cells expressing G $\alpha 0$ from either pCUP or pPGK.

A silent $B a m$ HI site was introduced into $S C G l$ to allow construction of the hybrid genes. Growth and mating were normal in both $s c g 1$ and SCGI cells expressing either pGAL-Scg or pGAL-Scg(B) (Fig. 3), consistent with the presumptively silent mutation. The Scg- $\alpha$ s, Scg- $\alpha \mathrm{i}$, and Scg- $\alpha 0$ hybrids efficiently complemented the scgl growth defect on either pGAL or pPGK vectors (Fig. 3 and Table 2), and the resulting cells were sterile. Again, the pPGK constructs were more effective than the pGAL constructs. The remaining hybrids, $\alpha s-S c g$ and $\alpha \mathrm{i}-\mathrm{Scg}$, expressed from either pGAL or pPGK, were unable to complement scgl.

Inhibition of pheromone response and mating by expression by $\mathbf{G} \alpha$ proteins. The sterility of $s c g l$ cells expressing heterologous or hybrid $\mathrm{G} \alpha$ proteins suggested that these proteins were able to interact with a downstream component of the pheromone response pathway to keep the pathway inactivated but were unable to interact with the pheromone receptors to elicit activation of the pathway in response to pheromone (see Discussion). If the heterologous and hybrid 
TABLE 2. Effects of $G \alpha$ protein expression

\begin{tabular}{|c|c|c|c|}
\hline Plasmid & $\begin{array}{l}\text { Growth } \\
\text { rate }^{a}\end{array}$ & $\begin{array}{c}\text { Inhibition of } \\
\alpha \text {-factor response }{ }^{b}\end{array}$ & $\begin{array}{l}\text { \% Inhibition } \\
\text { of mating }^{c}\end{array}$ \\
\hline PGK, GAL, CUP & - & - & 0 \\
\hline GAL- $\alpha s$ & $(+)$ & ++ & 35 \\
\hline CUP- $\alpha s$ & ++ & +++ & ND \\
\hline CUP- $\alpha$ s(sh) & ++ & +++ & ND \\
\hline PGK- $\alpha \mathrm{S}$ & +++ & +++ & 94 \\
\hline GAL- $\alpha i$ & - & $(+)$ & 0 \\
\hline CUP- $\alpha \mathrm{i}$ & $(+)$ & + & ND \\
\hline PGK- $\alpha \mathrm{i}$ & + & ++ & 47 \\
\hline CUP- $\alpha 0$ & - & - & 0 \\
\hline GAL-Scg- $\alpha S$ & ++ & ++ & 22 \\
\hline GAL-Scg- $\alpha i$ & +++ & +++ & 62 \\
\hline PGK-Scg- $\alpha s$ & +++ & +++ & 64 \\
\hline PGK-Scg- $\alpha i$ & +++ & ++++ & 100 \\
\hline PGK-Scg- $\alpha 0$ & +++ & ++++ & 80 \\
\hline PGK- $\alpha$ s-Scg & - & - & 0 \\
\hline PGK- $\alpha \mathrm{i}-\mathrm{Scg}$ & - & - & 0 \\
\hline GAL-Scg & ++ & - & 0 \\
\hline GAL-Scg(B) & +++ & - & 0 \\
\hline PGK-Scg & +++ & +++ & 86 \\
\hline
\end{tabular}

a Colony size was estimated for scgl segregants carrying the indicated plasmids (Fig. 3). Symbols (colony size): +++ , indistinguishable from $\mathrm{Scg}^{+}$; ++ , somewhat smaller; + , small; $(+)$, very small; - , identical to scgl null spores.

$b$ Assessed from the relative densities of growth within zones of inhibition surrounding $\alpha$-factor disks on test plates (Fig. 4). Symbols: ++++ , lack of a detectable inhibition zone; +++ , density 60 to $80 \%$ of lawn; ++ , density 30 to $60 \%$ of lawn; + , density 10 to $30 \%$ of lawn; $(+)$, several isolated colonies inside zone of inhibition; -, normal response.

c Assayed as described in Materials and Methods. Mating efficiency was normalized to matings of pairs of cells containing the PGK vector. ND, Not determined.

$\mathrm{G} \alpha$ proteins are able to interact with the downstream component in the presence of $S c g 1$, they should inhibit activation of the response pathway in the presence of pheromone, resulting in dominant inhibition of mating. We therefore tested $\alpha$-factor response and mating in wild-type SCGI strains expressing the various constructs.

Pheromone response was assayed by a plate assay for the inhibition of growth by $\alpha$-factor. Disks containing $\alpha$-factor produced clear, sharp-edged zones of growth inhibition on lawns of the SCG1 barl strain (DJ211-3-4) carrying the vectors without insert (Fig. 4). Cells containing constructs that complemented the scgl growth defect also showed zones of growth inhibition of similar sizes, but this inhibition was partial; i.e., a variable extent of growth was seen within the $\alpha$-factor zone. It has been suggested that growth within the zone of inhibition reflects more rapid recovery from growth inhibition, and a role has been proposed for $\operatorname{Scg} 1(G T P)$ in this adaptation phenotype $(6,7,21)$. Analysis of growth kinetics in scgl strains expressing the heterologous and hybrid $\mathrm{G} \alpha$ constructs (C. Mang and D. J. Tipper, unpublished data) suggested that the primary effect was on initiation of the response rather than on adaptation. We therefore interpret our assay as measuring inhibition of pheromone response. The relative levels of growth within the $\alpha$-factor zones were used to estimate the degree of inhibition (Table 2).

Expression of the same constructs also resulted in an inhibition of mating as determined by quantitative mating assays (Table 2). Inhibition was more effective when a particular construct was expressed in cells of both mating types rather than in only one of the mating pair; e.g., $94 \%$ inhibition of mating was seen when both a and $\alpha$ cells contained pPGK- $\alpha \mathrm{s}$, whereas inhibition was about $85 \%$ when cells of either mating type contained pPGK- $\alpha$ s and the strain of the opposite mating type contained the pPGK vector. The results summarized in Table 2 represent mating frequencies with strains of both mating types expressing identical constructs.

An excellent correlation between the ability to complement the scgl growth defect and the ability to inhibit pheromone response and mating in the $S C G l$ strain was seen (Table 2). Inhibition by $\mathrm{G} \alpha$ s was considerably greater than that by $\mathrm{G} \alpha \mathrm{i} 2$ expressed from the same construct, and $\mathrm{G} \alpha \mathrm{o}$ did not cause detectable inhibition. Inhibition was greater for a particular $\mathrm{G} \alpha$ protein expressed from $\mathrm{pPGK}$ or pCUP than when expressed from pGAL. The Scg- $\alpha \mathrm{i}$ and Scg- $\alpha$ o constructs had the strongest effects in all three assays of any construct tested.

Overexpression of SCG1 inhibited mating and response to $\alpha$-factor. Growth rates were normal in both scgl and SCGI cells expressing pPGK-Scg. However, while mating was normal in both $s c g l$ and $S C G l$ cells expressing pGAL-Scg, and expression of pGAL-Scg did not affect the $\alpha$-factor
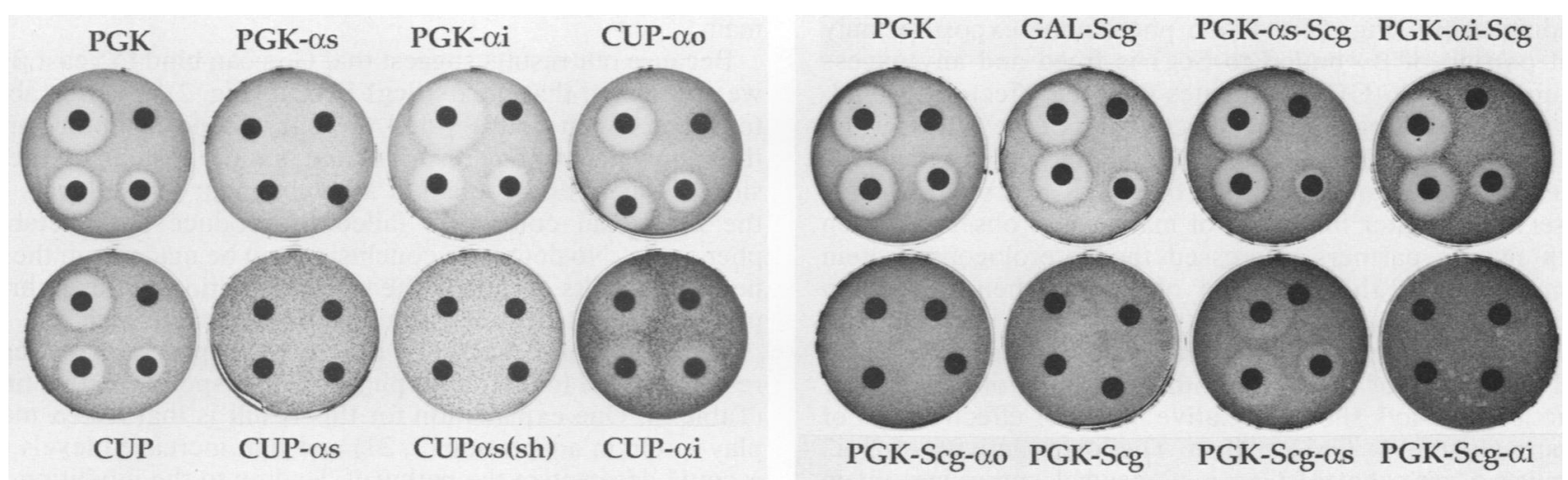

FIG. 4. Plate assays of $\alpha$-factor sensitivity. Cells of strain DJ211-3-4 (MATa SCGl barl), transformed with the indicated plasmids, were spread as a soft agar overlay onto SC-Ura or SC-Trp $\left(100 \mu \mathrm{M} \mathrm{Cu}^{2+}\right)$ buffered to $\mathrm{pH} 4.7$ and exposed to disks containing increasing quantities of $\alpha$-factor (see Materials and Methods). Zones are shown after $48 \mathrm{~h}$ at $30^{\circ} \mathrm{C}$. 
response in the wild-type a strain (DJ211-3-4) (Fig. 4), expression of pPGK-Scg efficiently inhibited $\alpha$-factor response in this strain. The test plates failed to show even faint zones of inhibition surrounding $\alpha$-factor disks (Fig. 4). Mating efficiency was also reduced by $86 \%$ when pPGK-Scg was expressed in both a and $\alpha$ cells (Table 2).

\section{DISCUSSION}

Scg1, the product of the yeast $S C G l$ gene, is a yeast $\mathrm{G} \alpha$ homolog involved in pheromone response and mating (10). It was previously shown that the expression of the long form of rat $\mathrm{G} \alpha \mathrm{s}$ in yeast was able to complement the growth and morphological defects resulting from null mutations in scgl (10). In this paper, we have extended this analysis to examine the effects of $\mathrm{G} \alpha \mathrm{s}(\mathrm{sh})$ (which lacks 15 amino acids present in the long form; 12), Goi2, Goo, and Scg1 and mammalian $\mathrm{G} \alpha$ hybrids. In addition, we have found that constructs able to complement the growth defect in an scgl mutant are also able to inhibit the response to $\alpha$-factor and mating when expressed in a wild-type $S C G l$ strain. Overexpression of Scg1 has a similar inhibitory effect.

These results are consistent with a simple model (Fig. 5A) for the role of the yeast $\mathrm{G} \alpha(\mathrm{Scg} 1), \mathrm{G} \beta$ (Ste4), and G $\gamma$ (Ste18) subunits in the activation of the pheromone response pathway. Genetic results $(1,24,32)$ indicate that $\beta \gamma$ functions downstream of $\alpha$ to activate the pathway, presumably by activating a downstream effector which is currently unidentified. In the absence of pheromone, $\alpha$ is presumed to bind GDP tightly and interact with $\beta \gamma$ to inhibit the pathway. In the presence of pheromone, the pheromone receptor interaction relieves this negative control by promoting GDP and GTP exchange on $\alpha$, resulting in dissociation of $\alpha$ from $\beta \gamma$; free $\beta \gamma$ then activates the pathway. In an scgl null mutant, free $\beta \gamma$ is present and constitutively activates the pathway (Fig. 5B), leading to $\mathrm{G} 1$ arrest and morphological alterations.

The ability of a mammalian $\mathrm{G} \alpha$ protein to complement the $s c g l$ growth defect suggests that the heterologous protein is able to interact with yeast $\beta \gamma$ as an analog of Scg1(GDP), thus preventing activation of the pathway (Fig. 5C). The resulting cells are sterile and unable to respond to pheromone, suggesting that the heterologous protein cannot interact functionally with the pheromone receptors. The ability of heterologous proteins to complement the $s c g 1$ growth defect correlates well with their ability to inhibit pheromone response and mating when expressed in wild-type SCG1 cells. This effect is also consistent with the model, because the heterologous or hybrid $\mathrm{G} \alpha$ protein competes with $\mathrm{Scg} 1$ for binding to $\beta \gamma$ (Fig. 5D). Upon pheromone exposure, only that portion of $\beta \gamma$ bound to Scg1 is freed and any excess heterologous G $\alpha$ (GDP) competes with the effector for binding to $\beta \gamma$, resulting in reduced activation of the pathway and reduced mating. The degree of inhibition should reflect the level of expression of the heterologous $\mathrm{G} \alpha$ protein, as was observed. Greater inhibition of mating was observed when both mating partners expressed the heterologous protein compared with the inhibition observed when expression occurred in only one of the two partners, because both mating partners must respond for mating to occur.

Comparison of $G \alpha s$ with other rat $G$ proteins by our functional assays showed relative levels of effectiveness of $\mathrm{G} \alpha \mathrm{s}=\mathrm{G} \alpha \mathrm{s}(\mathrm{sh})>\mathrm{G} \alpha \mathrm{i} 2>\mathrm{G} \alpha \mathrm{o}$. The lower activity of Gai2 compared with that of $\mathrm{G} \alpha \mathrm{s}$ is somewhat surprising, given that sequence conservation between $\mathrm{Scg} 1$ and $\mathrm{G} \alpha \mathrm{i} 2(10,23)$ is significantly higher than for Gos. Intriguingly, in vitro studies of $\mathrm{G} \alpha$ proteins $(2,12,16,27,28)$ indicate relative affinities of mammalian $\beta \gamma$ of $\mathrm{G} \alpha \mathrm{s}>\mathrm{G} \alpha \mathrm{i}>>\mathrm{G} \alpha \mathrm{o}$, which correlates with relative activities in our assays. Direct assays of $\mathrm{G}$ protein subunit interactions will be necessary to determine the relevance of this correlation.

The construction of Scg1 and mammalian G $\alpha$ hybrids was undertaken to analyze the role of various domains of $\mathbf{G} \alpha$ subunits (18) in the yeast signal transduction pathway. Fusions between $S C G I$ and rat $G \alpha$ cDNAs were made at a site within a highly conserved sequence of 32 amino acids predicted by analogy to ras (9) and EF-Tu (18) to comprise a critical component of the nucleotide-binding pocket of $\mathbf{G} \alpha$ proteins. The fusions, therefore, should be likely to retain normal structure in this region, and this region is unlikely to be involved in differential $\mathrm{G} \alpha$ functions. A $\mathrm{G} \alpha \mathrm{i}-\mathrm{G} \alpha$ s hybrid with the analogous fusion position has been shown to function in mammalian systems (19). The Scg1- $\alpha$, Scg1- $\alpha$ i2 and Scg1- $\alpha 0$ hybrids (Fig. 2) inhibited pheromone response and mating in an SCGI strain and allowed growth but not mating of the scgl null mutant. These results indicate sufficient conservation of structure between the yeast and mammalian $\mathrm{G} \alpha$ proteins to allow the function of appropriate domains in the hybrids and suggest that, like intact $\mathrm{G} \alpha$ s and G $\alpha$ i, these hybrid proteins can interact with $\beta \gamma$ but not with the pheromone receptors (Fig. 5). The sterility of scgl strains and inhibition of mating in $S C G 1$ strains expressing the hybrids are consistent with an essential role for the $\mathrm{C}$ terminus of $S c g 1$ in receptor interactions, as demonstrated in mammalian systems $(18,19,30)$. High-level expression of the N-terminal 330 amino acids of Scg1 by itself was without effect in our assays (data not shown), and a 22-amino-acid C-terminal truncation of Scg1 results in an scgl null phenotype (S. Hirsch, C. Dietzel, and J. Kurjan, unpublished data). Thus, the C-terminal region of Scg1 is essential to prevent activation of the pheromone response pathway by Scg1 in the absence of pheromone but can be replaced by heterologous $\mathrm{G} \alpha$ sequences.

The efficient activity of the Scg1- $\alpha \mathrm{i} 2$ and Scg1- $\alpha$ o hybrids, in contrast to that of the intact $\mathrm{G} \alpha \mathrm{i} 2$ and $\mathrm{G} \alpha \mathrm{o}$ proteins, suggests that the domain critical for interaction with $\beta \gamma$ lies within the N-terminal 330 amino acids of Scg1. These results, therefore, are consistent with data from mammalian systems that have indirectly implicated the amino termini of $\mathrm{G} \alpha$ proteins in $\beta \gamma$ interactions (18). The relative activities of the fusion proteins $(\operatorname{Scg} 1-\alpha \mathrm{i}=\mathrm{Scg} 1-\alpha \mathrm{o}>\mathrm{Scg} 1-\alpha \mathrm{s})$, unlike those of the intact proteins, do reflect sequence similarity to Scg1. The effectiveness of these fusions may depend, therefore, on the extent to which these heterologous C-terminal sequences interact appropriately with the N-terminal domain.

Because our results suggest that $G \alpha$ s can bind to yeast $\beta \gamma$, we speculated that the $\alpha s-S c g 1$ hybrid (Fig. 2) might be able to interact with both $\beta \gamma$ and the pheromone receptors and thus allow pheromone response and mating. Instead, expression of this hybrid protein at a level similar to the levels of the functional constructs failed to produce a detectable phenotype. No definitive conclusion can be made from these negative results, although the lack of function of this hybrid protein suggests that its structure may be abnormal.

We also found that very high level expression of Scg1 resulted in the inhibition of pheromone response and mating (Table 2). One explanation for this result is that free $\alpha$ may play a role in adaptation $(7,21)$ and that increased levels of $\alpha$ could desensitize the pathway, leading to the inhibition of pheromone response and mating. Alternatively, this result may reflect the importance of the balance between levels of the $G$ protein subunits for normal activation of the pathway. 


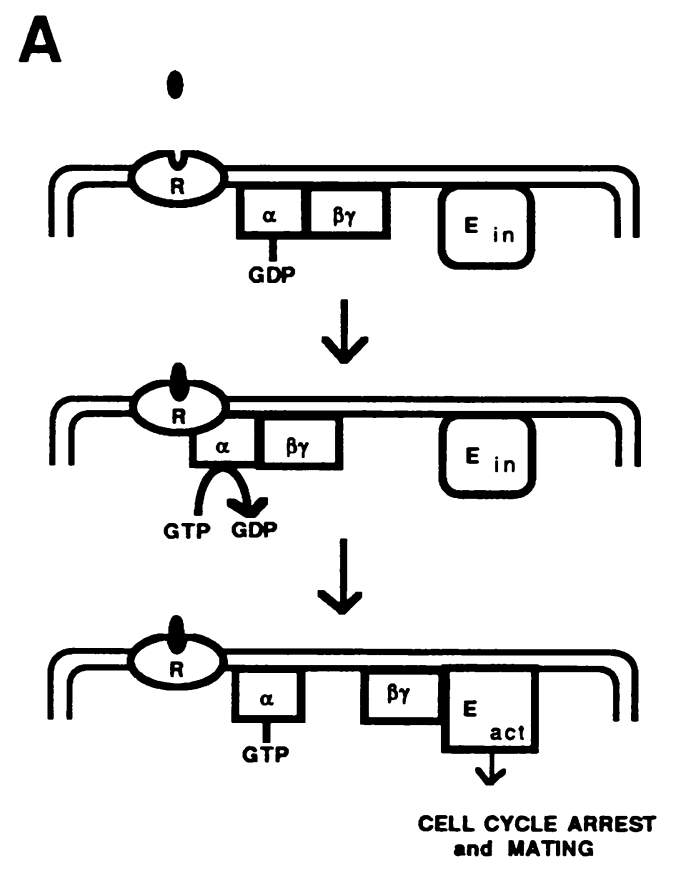

$\mathbf{B}$

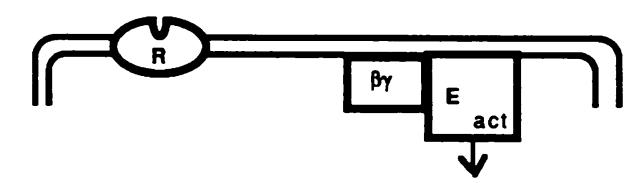

Bga1-null: CONSTITUTIVE CELL CYCLE ARREST

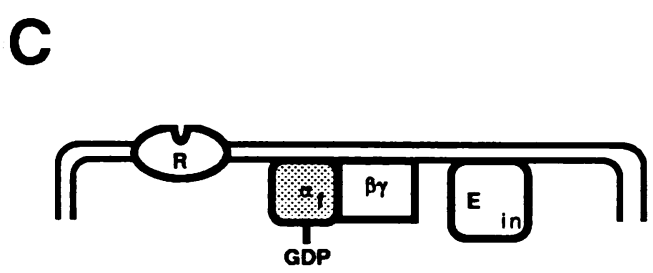

Beg1-nUll ABLE TO GROW BUT UNABLE TO

RESPOND TO PHEROMONE: STERILE

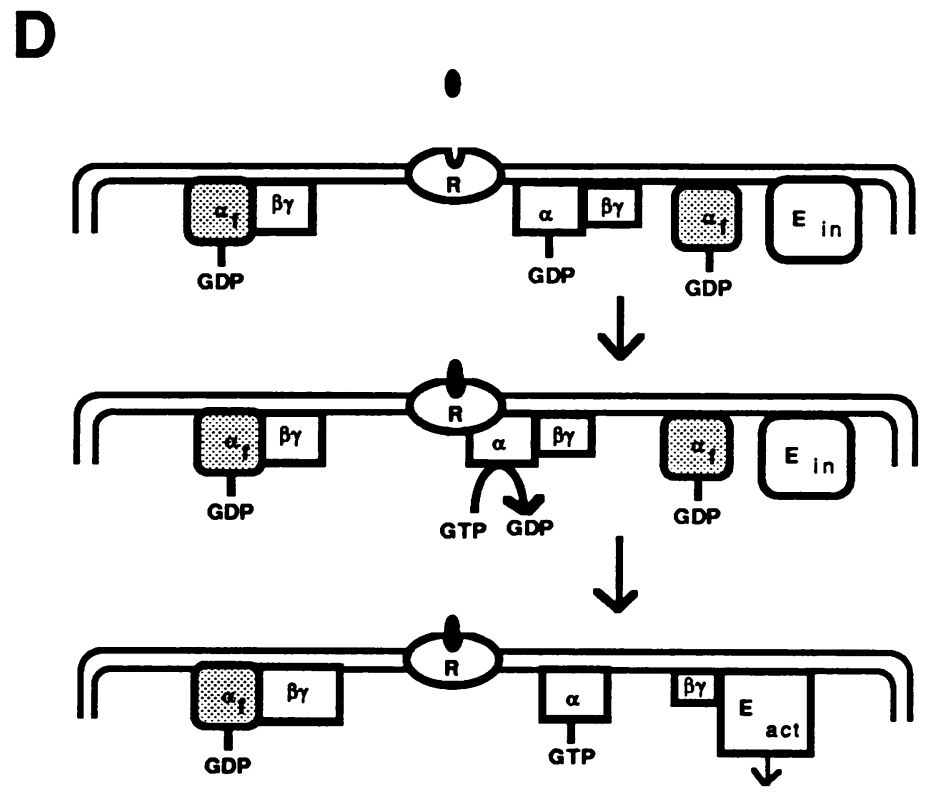

CELL CYCLE ARREST and MATING REDUCED in PROPORTION to QUANTITY of $G_{\alpha}$ and its AFFINTY for $\beta \gamma$

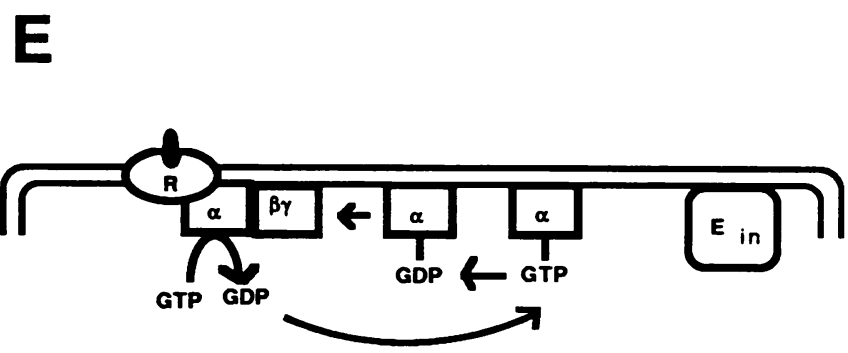

EXCESS OF Scg1 MASKS RESPONSE TO PHEROMONE

FIG. 5. Model for pheromone response pathway and effect of expression of heterologous or hybrid G $\alpha$ proteins. (A) In the absence of

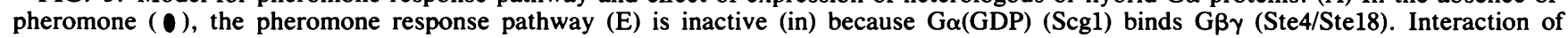
pheromone with its receptor induces a GDP-GTP exchange on $\mathrm{G} \alpha$, resulting in the release of $\mathrm{G} \beta \gamma$, which positively activates (act) the effector pathway, leading to cell cycle arrest and other aspects of the pheromone response. (B) In an $s c g l$ mutant, G $\beta \gamma$ is free, leading to constitutive activation of the pathway and cell cycle arrest. (C) A heterologous or hybrid G $\alpha$ protein $\left(\mathrm{G}_{\mathrm{f}}\right)$ complements the $s c g l$ growth defect in proportion to its ability to bind $\mathrm{G} \beta \gamma$. Because it cannot interact with the receptor, the pathway cannot be activated, so the cells are sterile. (D) When both $S \operatorname{cg} 1$ and a $G \alpha_{\mathrm{f}}$ are present, they compete for binding to $G \beta \gamma$. G $\alpha_{\mathrm{f}}$ remains bound to $G \beta \gamma$ after exposure to pheromone, and any excess $G \alpha_{\mathrm{f}}$ will compete with effector in binding $G \beta \gamma$ released by $\operatorname{Scg} 1(\mathrm{GTP})$, reducing activation of the pathway and mating. The degree of inhibition should be proportional to the level of $G \alpha_{f}$ and its affinity for $G \beta \gamma$. (E) In a strain greatly overproducing Scg1, conversion of $\operatorname{Scg} 1($ GDP) $\beta \gamma$ to dissociated Scg1(GTP) and $\beta \gamma$ by activated receptor is unaffected, but excess Scg1(GDP) is present and binds to freed $\beta \gamma$. The presumptive intrinsic GTPase activity of Scgl may result in the maintenance of Scgl(GDP) levels sufficient to keep the pathway inactivated.

It has recently been shown $(6,33)$ that increasing the level of Ste4 $(\beta)$ constitutively activates the pathway, while a simultaneous increase in the level of $\operatorname{Scg} 1(\alpha)$ prevents this constitutive activation. Our data suggest that greatly increasing $\alpha$ alone may result in the persistence of sufficient $\alpha$ (GDP) to complex the available $\beta \gamma$, even in the presence of a fully activated receptor (Fig. 5E). Increased levels of either receptor or $\beta \gamma$, therefore, might alleviate the inhibitory effect of increased $\alpha$ on the pheromone response.

\section{ACKNOWLEDGMENTS}

We thank J. Kevin O'Donnell for the preparation of the antiserum to the Gas fusion protein, Randall Reed and Gary Johnson for the generous gifts of cDNA clones, Duane Jenness for yeast strains, and Ted Wright for expert technical assistance. We thank Lorraine Marsh, Jeanne Hirsch, and Didi Robins for comments on the manuscript and Eliott Ross for pointing out the correlation for the $\mathrm{G} \alpha$ subunits between their activity in yeast and affinity for mammalian $\beta \gamma$. 
This work was supported by grants GM20755 and DK32520 (a pilot grant) to D.J.T. and by Public Health Service grant GM 40585 to J.K. from the National Institutes of Health.

\section{LITERATURE CITED}

1. Blinder, D., S. Bouvier, and D. D. Jenness. 1989. Constitutive mutants in the yeast pheromone response: ordered function of the gene products. Cell 56:479-486.

2. Bokoch, G. M., T. Katada, J. K. Northup, M. Ui, and A. G. Gilman. 1984. Purification and properties of the inhibitory guanine nucleotide-binding regulatory component of adenylate cyclase. J. Biol. Chem. 259:3560-3567.

3. Bostian, K. A., J. M. Lemire, and H. O. Halvorson. 1983. Physiological control of repressible acid phophatase gene transcripts in Saccharomyces cerevisiae. Mol. Cell. Biol. 3:839-853.

4. Bourne, H. R. 1989. G-protein subunits: who carries what message? Nature (London) 337:504-505.

5. Burkholder, A. C., and L. H. Hartwell. 1985. The yeast $\alpha$-factor receptor: structural properties deduced from the sequence of the STE2 gene. Nucleic Acids Res. 13:8463-8475.

6. Cole, G. M., D. E. Stone, and S. I. Reed. 1990 . Stoichiometry of G protein subunits affects the Saccharomyces cerevisiae mating pheromone signal transduction pathway. Mol. Cell. Biol. 10: 510-517.

7. Courchesne, W. E., R. Kunisawa, and J. Thorner. 1989. A putative protein kinase overcomes pheromone-induced arrest of cell cycling in Saccharomyces cerevisiae. Cell 58:2289-2297.

8. Cross, F., L. H. Hartwell, C. Jackson, and J. B. Konopka. 1988. Conjugation in Saccaromyces cerevisiae. Annu. Rev. Cell Biol. 4:430-458.

9. DeVos, A. M., L. Tong, M. V. Milburn, P. M. Matias, J. Jancarik, S. Noguchi, S. Nishimura, K. Miura, E. Ohtsuka, and S.-H. Kim. 1988. Three-dimensional structure of an oncogene protein: catalytic domain of human c-H-ras p21. Science 239: 888-893.

10. Dietzel, C., and J. Kurjan. 1987. The yeast SCGI gene: a $\mathrm{G} \alpha$-like protein implicated in the $a$ - and $\alpha$-factor response pathway. Cell 50:1001-1010.

11. Ecker, D. J., M. I. Khan, J. Marsh, T. R. Butt, and S. T. Crooke. 1987. Chemical synthesis and expression of a cassette adapted ubiquitin gene. J. Biol. Chem. 262:3524-3527.

12. Freissmuth, M., P. J. Casey, and A. F. Gilman. 1989. G proteins control diverse pathways of transmembrane signalling. FASEB J. 3:2125-2131.

13. Hagen, D. C., G. McCaffrey, and G. F. Sprague, Jr. 1986. Evidence the yeast STE3 gene encodes a receptor for the peptide pheromone a-factor: gene sequence and implication for the structure of the presumed receptor. Proc. Natl. Acad. Sci. USA 83:1418-1422.

14. Jahng, K.-Y., J. Ferguson, and S. Reed. 1988. Mutations in a gene encoding the $\alpha$ subunit of a Saccharomyces cerevisiae G protein indicate a role in mating pheromone signaling. Mol. Cell. Biol. 8:2484-2493.

15. Jones, D. T., and R. R. Reed. 1987. Molecular cloning of five GTP-binding protein cDNA species from rat olfactory neuroepithelium. J. Biol. Chem. 262:14241-14249.

16. Katada, T., G. M. Bokoch, M. D. Smigel, M. Ui, and A. G. Gilman. 1984. The inhibitory guanine nucleotide-binding regulatory component of adenylate cyclase: subunit dissociation and guanine nucleotide-dependent hormonal inhibition. J. Biol. Chem. 259:3586-3595.

17. Kim, D., L. D. Lewis, L. Graziadei, E. J. Neer, D. Bar-Sagi, and D. E. Clapham. 1989. G-protein $\beta \gamma$-subunits activate the cardiac muscarinic $\mathrm{K}^{+}$channel via phospholipase $\mathrm{A}_{2}$. Nature (London) 337:557-560.

18. Masters, S. B., R. M. Stroud, and H. R. Bourne. 1986. Family of G protein $\alpha$ chains: amphipathic analysis and predicted structure of functional domains. Protein Eng. 1:41-54.

19. Masters, S. B., K. A. Sullivan, R. T. Miller, B. Beiderman, N. G. Lopez, J. Ramachandran, and H. R. Bourne. 1988. Carboxyl terminal domain of $\mathrm{G}_{\mathrm{s} \alpha}$ specifies coupling of receptors to stimulation of adenylyl cyclase. Science 241:448-451.

20. Mellor, J., M. J. Dobson, N. A. Roberts, M. F. Truite, J. S. Emtage, S. White, P. A. Lowe, T. Patel, A. J. Kingsman, and S. M. Kingsman. 1983. Efficient synthesis of enzymatically active calf chymosin in $S$. cerevisiae. Gene 24:1-14.

21. Miyajima, I., K.-I. Arai, and K. Matsumoto. 1989. GPAI $I^{\text {Val-50 }}$ mutation in the mating-factor signaling pathway in Saccharomyces cerevisiae. Mol. Cell. Biol. 9:2289-2297.

22. Miyajima, I., M. Nakafuku, N. Nakayama, C. Brenner, A. Miyajima, K. Kaibuchi, K. Arai, Y. Kaziro, and K. Matsumoto. 1987. GPA1, a haploid-specific essential gene, encodes a yeast homolog of mammalian $G$ protein which may be involved in mating factor signal transduction. Cell 50:1011-1019.

23. Nakafuku, M., H. Itoh, S. Nakamura, and Y. Kaziro. 1987 Occurrence in Saccharomyces cerevisiae of a gene homologous to the cDNA coding for the $\alpha$ subunit of mammalian $G$ proteins. Proc. Natl. Acad. Sci. USA 84:2140-2144.

24. Nakayama, N., Y. Kaziro, K.-I. Arai, and K. Matsumoto. 1988. Role of STE genes in the mating factor signaling pathway mediated by GPAl in Saccharomyces cerevisiae. Mol. Cell. Biol. 8:3777-3783.

25. Nakayama, N., A. Miyajima, and K. Arai. 1985. Nucleotide sequences of STE2 and STE3, cell type-specific sterile genes from Saccharomyces cerevisiae. EMBO J. 4:2643-2648.

26. Neer, E. J., and D. E. Clapham. 1988. Roles of G protein subunits in transmembrane signalling. Nature (London) 333: 129-134.

27. Sternweis, P. C. 1986 . The purified $\alpha$ subunits of $G_{o}$ and $G_{i}$ from bovine brain require $\beta \gamma$ for association with phospholipid vesicles. J. Biol. Chem. 261:631-637.

28. Sternweis, P. C., and J. D. Robishaw. 1984. Isolation of two proteins with high affinity for guanine nucleotides from membranes of bovine brain. J. Biol. Chem. 259:13806-13813.

29. Stryer, L., and H. R. Bourne. 1986. G proteins: a family of signal transducers. Annu. Rev. Cell Biol. 2:391-419.

30. Sullivan, K. A., R. T. Miller, S. B. Masters, B. Beiderman, W. Heideman, and H. R. Bourne. 1987. Identification of receptor contact site involved in receptor-G protein coupling. Nature (London) 330:758-762.

31. Tong, L., A. M. deVos, M. V. Milburn, J. Jancarik, S. Noguchi, S. Nishimura, K. Miura, E. Ohtsuka, and S.-H. Kim. 1989. Structural differences between a ras oncogene protein and the normal protein. Nature (London) 337:90-93

32. Whiteway, M., L. Hougan, D. Dignard, D. Y. Thomas, L. Bell, G. C. Saari, F. J. Grant, P. O'Hara, and V. L. MacKay. 1989. The STE4 and STE18 genes of yeast encode potential $\beta$ and $\gamma$ subunits of the mating factor receptor-coupled $\mathrm{G}$ protein. Cell 56:467-477.

33. Whiteway, M., L. Hougan, and D. Thomas. 1990. Overexpression of the STE4 gene leads to mating response in haploid Saccharomyces cerevisiae. Mol. Cell. Biol. 10:217-222.

34. Young, J. F., U. Desselberger, P. Palese, B. Ferguson, A. R. Shatzman, and M. Rosenberg. 1983. Efficient expression of influenza virus NSI nonstructural proteins in E. coli. Proc. Nat. Acad. Sci. USA 80:6150-6109. 